\title{
Stellar Spirals in Triaxial Dark Matter Halos
}

\section{Shaoran $\mathrm{Hu}$ and Debora Sijacki}

Institute of Astronomy and Kavli Institute for Cosmology, University of Cambridge, Madingley Road, Cambridge CB3 0HA, email: sh759@ast.cam.ac.uk

\begin{abstract}
Two-armed grand-design spirals may form if the shape of its dark matter halo changes abruptly enough. The feasibility of such a mechanism is tested in realistic simulations. The interplay of such externally-driven spirals and self-induced transient spirals is then studied. Subhaloes are also found to lead to transient grand-design spiral structures when they impact the disk.
\end{abstract}

Keywords. galaxies: spiral, galaxies: kinematics and dynamics, galaxies: halos

Introduction. Spiral structures can form either as a response to external perturbations or due to self-gravity. In cosmological simulations, the shape of the haloes changes violently over time. In this work we investigate its influence on the formation of spiral structures, and its interplay with the self-gravity of the disk.

Method. As described in detail in Hu \& Sijacki (2016), we employ N-body simulations of live disks using Gadget-3 (Springel et al. 2005). The triaxial dark matter haloes are modeled analytically with its triaxiality varying with radius and time.

Results. Our main findings are:

- Triaxial dark matter haloes lead to grand-design spirals if and only if their shape changes abruptly enough. Once formed, such spirals can persist for several Gyrs. The underlying mechanism of such spirals are kinematic density waves (Lindblad 1963).

- As shown in Fig. 1, a halo similar to Aq-B halo in the Aquarius simulation (Springel et al. 2008) can lead to spiral structures, demonstrating that such a mechanism is realistic.

- When the swing amplification is strong, flocculent spirals formed by swing amplification of Poisson noise and of the grand-design spirals finally dominate the disk.

- Substructures in the dark matter halo that hit the disk may also lead to transient, single-armed, grand-design spiral structures.
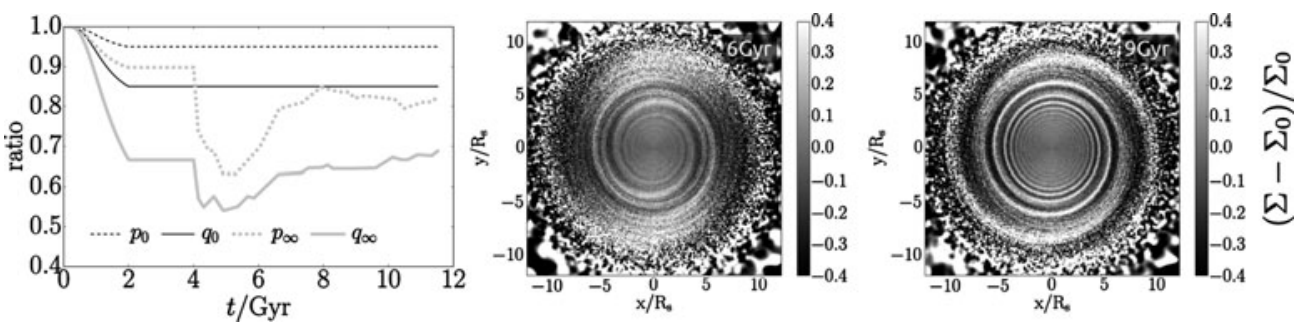

Figure 1. Left: ratio of the three axes $(p=b / a, q=c / a)$ of the halo at the innermost and the outermost region, the latter changing over time following Vera-Ciro et al. (2011). Middle \& right: difference of surface density $\Sigma$ to the initial condition $\Sigma_{0}$ where prominent spirals form.

\section{References}

Hu, S. \& Sijacki, D. 2016, MNRAS, 461, 2789

Springel, V., Di Matteo, T., \& Hernquist, L. 2005, MNRAS, 361, 776

Lindblad, B. 1963, Stockholms Observatoriums Annaler, 22, 5

Springel, V., Wang, J., Vogelsberger, M. et al. 2008, MNRAS, 391, 1685

Vera-Ciro, C. A., Sales, L. V., Helmi, A. et al. 2011, MNRAS, 416, 1377 